\title{
Nonintentional analogical inference in text comprehension
}

\author{
Samuel B. Day and Dedre Gentner \\ Northwestern University, Evanston, Illinois
}

\begin{abstract}
We present findings suggesting that analogical inference processes can play a role in fluent comprehension and interpretation. Participants were found to use information from a prior relationally similar example in understanding the content of a later example, but they reported that they were not aware of having done so. These inference processes were sensitive to structural mappings between the two instances, ruling out explanations based solely on more general kinds of activation, such as priming. Reading speed measures were consistent with the possibility that these inferences had taken place during encoding of the target rather than during the later recognition test. These findings suggest that analogical mapping, though often viewed as an explicit deliberative process, can sometimes operate without intent or even awareness.
\end{abstract}

In making our way through the world, we are confronted with a constant stream of information - far too much to encode exactly. One of our most fundamental tasks is therefore simply to make sense of the things we encounter, organizing incoming information in relation to our expectations and prior experience. For instance, witnessing a scene with a woman in an elaborate white gown may cause us to classify her as a bride, which leads to the activation of our schema for a traditional wedding ceremony. This, in turn, allows us to determine the roles of other individuals (e.g., the man next to her is her groom, the person facing them is their minister) and to make countless additional attributions and inferences about the people and events involved. All of this is accomplished so quickly and so effortlessly that we tend to underestimate the complexity of the cognitive processes that are demanded.

It seems uncontroversial that categorization and schema activation may contribute to the fast, nondeliberative assignment of meaning to the world. Another process that has not generally been considered in this way is analogy, which brings to bear specific prior events or episodes.

In analogical processing, new information about an object or event may be inferred on the basis of structural similarities to a better-understood instantiated system. One classic example is Rutherford's model of the atom, which was based on knowledge about the solar system (see Gentner, 1983). By delineating a few common relations shared by the two systems, Rutherford was able to make informed speculations about additional properties and spatial and causal relationships within the atom. Additional research has highlighted the important role that analogy continues to play in contemporary scientific discoveries (see, e.g., Dunbar, 1995, 1999; Gentner, 2002; Thagard, 1992). As these and countless other examples demonstrate, analogy provides an invaluable tool for reasoning about poorly understood situations, solving difficult problems, and making plausible inferences about unknown properties, behaviors, and characteristics (Gentner \& Markman, 1997; Holyoak \& Thagard, 1995; Hummel \& Holyoak, 1997).

Although a substantial body of research exists looking at this "traditional" sort of analogy use, it is inviting to consider whether the same analogical processes may also be involved in the far more routine task of organizing and interpreting our daily experiences. Intuitively, it seems that analogies with our prior experience could contribute to our fluency in processing current situations, even when such analogies are not overtly noticed or intentionally pursued. But such a use of analogy poses some challenges to previous research, which has (explicitly or implicitly) treated analogical processing as an inherently slow, analytical activity.

In the experiments presented in this article, we examined this possibility and found evidence supporting the use of analogy in structuring and understanding novel information. Specifically, when given a written passage in which certain facts were left unstated or ambiguous, individuals were likely to make interpretations that paralleled structural information from a previously read, analogous scenario. In contrast with the great majority of existing research, as well as with some common conceptions about analogy use, this interpreting was done in the absence of directive instruction, without any explicit goal other than comprehension, and strikingly, without apparent participant awareness of analogical inference having occurred.

\section{Analogy and Analogical Processing}

Formally, an analogy is a kind of similarity in which the same system of relations holds across different objects 
(Gentner, 1983). Cognitive processing of analogies has been a fertile and productive area for research over the last two decades, and there is substantial consensus on the fundamental processes involved (Gentner, 2003; Gentner, Holyoak, \& Kokinov, 2001; Gentner \& Markman, 1997; Holyoak \& Thagard, 1989; Hummel \& Holyoak, 1997; Kokinov \& French, 2003). A key characteristic of analogical theories is the emphasis on structured representations that specify the relations between elements.

For example, in structure-mapping theory (Forbus, Gentner, \& Law, 1995; Gentner, 1983, 2003; Gentner \& Markman, 1997), the comparison processes act to achieve a maximal structurally consistent alignment between two representations. Structural consistency entails that the correspondences between the elements and relations in two representations must satisfy one-to-one correspondence (an element in one representation may be mapped to at most one element in another) and parallel connectivity (if two predicates correspond, their arguments must also correspond). Once a structural alignment has been established, candidate inferences are projected. These are additional elements that are connected to the common system in the base (or source) structure but are not yet present in the target (Clement \& Gentner, 1991; Markman, 1997). Importantly, in structure-mapping, such inferences arise automatically via a structural pattern completion process.

\section{Analogy and the Present Experiments}

The existing findings on analogical mapping processes have mostly been derived from studies in which participants were explicitly provided with both the base and the target and were asked to identify commonalities, correspondences, or potential inferences between the two (e.g., Bowdle \& Gentner, 1997; Markman, 1997; Spellman \& Holyoak, 1996). In the other most common paradigm, participants were given a set of problems and then later asked to solve a problem that was analogous to a prior problem, although participants were not told of this connection (e.g., Gick \& Holyoak, 1980, 1983; Loewenstein, Thompson, \& Gentner, 1999; Novick, 1988; Ross, 1984, 1987). In other words, analogical inference has generally been examined in situations in which the analogy was explicitly pointed out to participants, the inferences were made in the service of an experimentally directed goal, or both.

But what about the possibility of spontaneous, nonintentional inference? It is generally acknowledged that we make use of prior, structured experience in understanding new situations, and an intuitive appraisal of our processing in these instances suggests that these inferences are often outside the sphere of awareness or active deliberation. Of course, this phenomenon could be restricted to cases in which repeated exposure to a kind of event has led to a generalized schema. Our question, in contrast, was whether analogical transfer (i.e., mapping from a single prior instance) could occur in a spontaneous, nondeliberative way.

To qualify as analogical transfer, such inferencing must be shown to involve the same kinds of sophisticated alignment and mapping processes that are involved in intentional analogical inference. To examine these issues in the present experiments, we employed a different approach from the ones adopted in prior studies of analogy. Our participants were given a series of short narrative passages to read and were simply told that they would later be asked questions about their content. Some of the stories were designed to be analogous to earlier passages, but with some crucial piece of information missing or ambiguous. Our goal was to determine whether individuals would spontaneously make use of this analogy in their interpretation of the later story's meaning, without necessarily being aware of doing so. The domain of text processing seems well suited to exploring these kinds of effects, since reading a text passage typically involves drawing inferences that fill in unstated but necessary information (see, e.g., Glenberg, Meyer, \& Lindem, 1987; Graesser, Singer, \& Trabasso, 1994; Kintsch, 1988).

Our experiments differed from most prior work on analogy in two important ways. First, participants had no explicit goal that would require them to draw on one example for potential information about another; they were simply given passages to read and remember. The participants' primary objective, then, was merely to understand what they were reading. Second, in most prior research, participants were aware that analogical inference was taking place. We, on the other hand, designed the methodology of the present experiments in order to examine inferences that are made spontaneously, perhaps even without awareness. Rather than asking participants to consciously generate or rate the plausibility of inferences or to actively transfer a principle to a new analogous situation, we simply asked our participants to remember the stories. We then gave them a recognition task that measured the extent to which they had incorporated information from the base into their representations of the target, influencing their beliefs about what was actually stated. In sum, the goal of the present experiments was to determine whether analogy may be counted among those "invisible" processes that can contribute to rapid, nondeliberative tasks, such as comprehending text.

\section{Related Research in Analogy}

Some research exists that is relevant to these issues. In an innovative and interesting set of experiments, Blanchette and Dunbar (2002) examined analogy's role in online interpretation. They gave participants descriptions of a target issue (e.g., legalizing marijuana) followed in the same passage by a situation that was identified as analogous (e.g., ending Prohibition). On a subsequent recognition test, participants often misidentified analogical inferences as facts actually presented about the target. Perrott, Gentner, and Bodenhausen (2005) found similar effects even when the inferences ran contrary to participants' (previously assessed) attitudes toward the target. In another set of experiments, Moreau, Markman, and Lehmann (2001) demonstrated the influence of analogy in interpreting new information about a novel domain. They found that in making inferences about digital cameras (which were very new at the time), the choice of base domain (film cameras vs. scanners) significantly altered how information about the target was structured by participants. However, in all of these lines of research, the analogy between the two examples was made explicit for participants. 
Catrambone (2002) presented another set of related findings, in which he demonstrated that analogical similarity can play a significant role in reminding. In these experiments, which used narrative passages derived from the "Karla the Hawk" stories (Gentner, Rattermann, \& Forbus, 1993), he found that a later passage was more likely to lead to reminding of an earlier passage as the number of relational commonalities between the two was increased. This is particularly interesting in light of the large body of research showing difficulties in relational reminding (see the General Discussion below). He also presented reading speed data suggesting that these remindings led to structural mappings between the two passages, consistent with the present proposal. Unlike the present set of experiments, however, those were not aimed at capturing implicit inference processes; no evidence was given regarding inferencing of new content or of changes in participants' representations of the target as a function of those remindings.

These findings provide evidence that analogy can provide a basis for representational structuring. However, the question of whether analogical processes may actually be used as a vehicle for the spontaneous, nonintentional structuring of new information has as yet received little or no attention.

Two further bodies of research are also relevant here: schema theory, which addresses the role of existing knowledge on new information, and theories of text comprehension, which examine how input information is integrated into a globally coherent structure.

\section{Schemas}

Schemas are abstract knowledge structures that are hypothesized to mediate the interaction between existing knowledge and new experiences. Although the literatures on schemas and analogy are largely independent, the two ideas have much in common. Both represent ways in which existing knowledge may be used to inform situations that are less well understood, and both rely on structured representations to do so. And, just as an analogy aligns the relational structures of two instances, a schema defines the relationships between variable placeholders and leads to mappings between these slots and items in the environment. The primary difference is that schemas are knowledge structures that have been abstracted over several episodes, whereas an analogy is a mapping from one particular prior instance. However, this distinction may be a matter of degree rather than a truly qualitative difference. In fact, many have suggested that both schematic knowledge and general conceptual knowledge should be thought of in a continuum between concreteness and abstraction (Anderson, 1984; Bowdle \& Gentner, 2005; Gentner \& Medina, 1998; Medin \& Ross, 1989; Rumelhart \& Ortony, 1977). Gentner and Medina proposed that schemas and other abstractions are often derived via a process of repeated analogizing over instances (see also Cheng \& Holyoak, 1985).

Given these commonalities, it makes sense to look to the schema literature for insights about analogical processing, and indeed the kinds of effects that we are investigating have been suggested in schema research. For example, schemas have been shown to influence both how people structure incoming information (see, e.g., Anderson, Spiro, \& Anderson, 1978; Bransford \& Johnson, 1972) and how new information is added during the encoding of a situation (e.g., Steffensen, Joag-Dev, \& Anderson, 1979). Additionally, there are results suggesting that these schema-driven effects can take place outside of conscious awareness (e.g., Anderson, Reynolds, Schallert, \& Goetz, 1977). In the present experiments, we examined whether these sorts of effects are specific to abstracted schematic knowledge structures or whether they can also operate through analogy to a single concrete instance.

\section{Summary}

The goal of this research was to investigate an important open question: Can a single prior instance influence how a new episode is understood, and if so, does it do so by using a structurally sensitive mapping process, as in analogy? The finding that spontaneous analogical retrieval and structural mapping can be accomplished via fast, nonintentional processing would represent a major new arena for analogical phenomena.

\section{Our Experiments}

In three experiments, we examined whether participants' interpretations of a passage would be influenced by structural information from a recently read analogous passage. Each of the experiments involved reading a series of brief scenarios, with some of the later stories being relationally similar to stories earlier in the set. In all cases, there were two versions of each base passage, with some piece or pieces of information varied between them. The later target passages left this particular element either unstated or ambiguous, leaving it open to more than one interpretation. In the first two experiments, yes-or-no questions about the target passages were used to determine whether participants had interpreted those stories in a manner consistent with the structure of the base passages they had read. In Experiment 2, we also varied the structural role of relevant concepts in the base to test whether the target inferences might have stemmed from simple semantic activations rather than from structural commonalities. In these first two experiments, we used a later recognition task; therefore, the possibility remained open that any observed inferences in the target were taking place during the test phase rather than during encoding, contrary to our suggestion. To explore this possibility, in Experiment 3 , we used a reading time measure to monitor online processing of the target passages. These two approaches complement one another, allowing us to examine both the content and the timing of participants' inferences.

\section{EXPERIMENT 1}

Our primary motivation was to test whether individuals may, in a nonintentional way, make use of analogical alignment and inferences from a single prior instance in structuring novel information. In Experiment 1, we established a simple paradigm for examining this issue; in Experiments 2 and 3, we attempted to rule out some possible counterexplanations. 


\section{Method}

Participants. The participants were 20 undergraduate students at Northwestern University who participated for class credit.

Materials and Procedure. The materials consisted of a series of narrative passages and a set of questions about their content. The story set contained nine passages: two base passages, two target passages, and five filler stories. The average length of the passages was 174 words $(S D=76)$. Above each story was a brief (two- to threeword) title describing some salient feature of the passage, such as the setting (e.g., "Jungle Expedition," “van Houton's Funeral”).

There were two versions of each base passage, varying in some meaningful piece of information. This variation, referred to here as the key fact, involved an average change of nine words between versions, leaving the great majority of the between-participants passages identical. The target passages left the information that was analogous to the key fact either unstated or ambiguous, and they were designed to be equally comprehensible with either of the possible interpretations inferable from the base. Which version of the base was read was varied randomly between participants, resulting in two groups (10 per group). All participants read the same filler and target passages. Participants were given the story set along with the following instructions: "Please read each of the following passages quickly but thoroughly. Later, you will be asked to answer a few questions about them."

As an example, one of the base passages described a wealthy widow who had died under suspicious circumstances (see Appen$\operatorname{dix}$ A). In one version of the base, the niece, who lived in another city, dutifully flew to her aunt's home upon the announcement of her death. In the other version, the niece had been in the same city as her aunt but mysteriously left town when the death was announced. The two versions of the base were otherwise identical and contained considerable additional elaborative information. The target passage closely paralleled the bases, in terms of both relational structure and content similarity, except for an ambiguity relating to the key fact. For example, the target passage described the mysterious death of a wealthy older man and then simply stated that the man's nephew, George, "immediately bought a ticket and flew to Rio de Janeiro" upon the death's announcement. This leaves unstated whether it is a flight to or from his uncle's home, or neither. The question was whether participants would interpret the nephew's actions in a manner consistent with an inference from the particular version of the base passage they had read.

The participants read the nine stories at their own pace, usually requiring 5-10 $\mathrm{min}$ for the set. Once finished, they performed an unrelated filler task (which involved judging similarities between animated scenes of motion) that took approximately $20 \mathrm{~min}$ to complete, and they then responded to a set of test items about the stories. The test items were given with the following instructions: "Please circle Yes or No to indicate whether each of the following facts was stated in the passages you read earlier. To assist you, the title of the relevant story is given in italics before each fact."

The question set contained 18 items, 2 per passage. For each of the base and filler stories, there was one fact that had actually been presented in the passage and one fact that had not, so that yes and no responses were correct equally often. For the target passages, there was one fact consistent with the potential inference given by each of the two possible bases. For instance, the two items for the target described above were the following:

George had been in the same city as his uncle, but left town when the death was announced.

George was among those who came into town for Alexander van Houton's funeral.

If participants had imported the relational structure from the base passage to the target, they would be more likely to falsely recognize statements that were consistent with an inference from the base version they had read, relative to the version not read. Note that these sentences served as their own controls, since each could be either consistent or inconsistent, depending on the given base. Thus, there were 4 relevant items ( 2 for each of the targets) and 14 filler items that served as memory checks. All participants responded to the same 18 items, and the order of these items was randomized for each individual.

Following these test items, participants were given a set of openended questions designed to assess their awareness of the processes involved in the inference and interpretation. These included some general questions about the participants' overall impressions of the stories and questions that more specifically addressed their awareness of the passages' similarity and ambiguity.

\section{Results and Discussion}

For each participant, a proportion of yes responses was calculated separately for base-consistent and baseinconsistent items. A one-way ANOVA was performed using these proportions as a within-subjects factor. This test showed that participants were significantly more likely to respond that an idea had been stated in the target passage when that idea could be inferred from the base passage they had $\mathrm{read}[F(1,19)=22.94, p<.001]$. On average, participants believed they had read an idea in the target $72.5 \%$ of the time when that idea was baseconsistent, versus only $25 \%$ of the time when that same idea was base-inconsistent. An items analysis was also significant, using base consistency as a within-items factor $[F(1,3)=98.46, p<.01]$, and for all target items, the number of positive responses was greater when that item was consistent with the base passage. Because of the relatively small number of test items, we also conducted a $\operatorname{Min} F^{\prime}$ analysis, as a more conservative test. This analysis also showed a significant effect $\left[\operatorname{Min} F^{\prime}(1,21.5)=18.61\right.$, $p<.01]$.

In terms of evaluating participant awareness of the inference process, two of the open-ended questions were relevant. When asked whether they had noticed similarities between any of the stories, 90\% of the participants responded that they had, frequently citing specific examples. This is not surprising, given the level of overall similarity between base-target pairs; in fact, a failure to recognize these similarities would probably have reflected inattentive reading. More importantly for the present purposes, though, most participants appeared unaware of having used any of these prior stories to help interpret a later one. When asked "Did you feel that each of the passages was completely understandable on its own, or did you find yourself referring to previous stories in order to understand later ones?" $80 \%$ of participants stated that all passages were completely understandable on their own.

The results are consistent with the claim that individuals may use a single prior instance as a source for nonintentional inference based on structural commonalities. The pattern of inferences is what would be expected if participants were structurally aligning the two representations and drawing inferences about the target from relationally similar aspects of the base. Participants' responses that the inferred information had actually been stated in the target story suggest that these inferences were not deliberately considered and evaluated, but rather were spontaneously incorporated into the target representations as they were being created. 
The outcome of Experiment 1 offers support for the possibility of nonintentional analogical inference. However, there are several alternative explanations that could also account for these results. For instance, participants could have confused or conflated the base and target stories in memory, or recognized an abstracted relational structure rather than the target itself. They could also have misunderstood the instructions when indicating that information was stated when it was merely implied, or even responded to a perceived demand characteristic. We addressed these issues in Experiment 3. Another possibility is that the influence of the base on the target occurred not through aligning common relational structures, but through a simpler kind of semantic activation. In our second experiment, we examined this last issue.

\section{EXPERIMENT 2}

We are suggesting that a process of structural alignment between the base and target passages leads to the importing of some information from one to the other by a process of structural pattern completion (Clement \& Gentner, 1991; Markman, 1997). This would involve aligning the passages' represented structures and carrying over connected assertions from the base to the target. Structural commonalities would play a fundamental role in this process. It is possible, however, that the influence occurs in a much simpler way that does not rely on analogical mapping. For instance, the presence of information in the base could simply serve to increase its activation, making this information more fluent and accessible during the process of comprehending the target.

Ross and Bradshaw (1994) demonstrated that very simple semantic associations can have a profound impact on interpretation. Their experiments made use of passages that could be interpreted in multiple ways, such as the stories by Anderson et al. (1977), which could be plausibly interpreted as describing a wrestling match or a jailbreak (with sentences such as "The lock which held him was strong, but he thought that he could break it"). Prior to reading these stories, participants had read passages mentioning the themes that could be used to comprehend the ambiguous story, and these themes had been arbitrarily associated with some other concept. For example, one earlier story mentioned that a character enjoyed reading Shakespeare and watching wrestling, and another story described a writer for the Delaware Daily News who had once covered a story about a jailbreak. After this brief reference, the themes were not mentioned again. The ambiguous passage included an early sentence stating that its character had begun his day by reading something - either Shakespeare or the Delaware Daily News. There was a strong and significant tendency for readers to interpret this new passage according to the theme that had previously been associated with the mentioned reading material.

Simple associative effects such as this could potentially account for the base-consistent inferences in Experiment 1 . The similarities between base and target stories seem more than adequate to encourage reminding (as corroborated by our posttest questions). Once activated, the contents of the base could represent an unstructured set of semantic activations that simply added to the fluency of certain ideas, making them more likely to participate in interpretive processing in general. If this were the case, no mapping of relational structure would be necessary.

In Experiment 2, we examined this possibility by using base passages that each contained both of the key facts that could be used to interpret the ambiguous portion of the target. One of the facts played the same structural role in the base as the ambiguous statement did in the target; the other occurred in a different part of the base. If the earlier effects were driven by structural alignment, in this experiment we should see inferences derived from the fact in a structurally corresponding role.

\section{Method}

Participants. The participants were 20 undergraduate students at Northwestern University who participated for class credit.

Materials and Procedure. The materials again consisted of a set of narrative passages and a set of questions about their content. The story set included nine passages - two base-target pairs and five filler stories - presented in the following order: filler, Bases 1 and 2, two fillers, Target 1, filler, Target 2, filler. The average story length was 182 words $(S D=84)$. Each story was again given a brief descriptive title.

As in Experiment 1, there were two versions of each base passage. In this experiment, however, each base contained both of the concepts that could be used to interpret the ambiguous portion of the target, but in different locations depending on condition. In each version of the base, one of the key facts was in a position relationally similar to the target's ambiguous element, the other was not.

For example, one of the base passages describes a herpetologist who is studying animal life in the jungles of Peru (see Appendix B). The area is said to have only recently been opened for exploration as a result of a new, nearby industrial refinery that had increased travel accessibility. The scientist notices a lizard that is similar to specimens he has seen before, but that has some unusual physical characteristics, such as darker coloration and more slender limbs. In one version of the passage, he is excited to realize that he has discovered an unknown species; in the other version, he is disturbed to realize that he has found a mutation resulting from pollution. In both of these versions, the alternate idea is mentioned in a later part of the passage, which describes the scientist's subsequent work. The "undiscovered species" version goes on to describe how he is later hired to "investigate the impact of pollution on animal growth and development," whereas in the "mutation" version he is hired to "search for previously undiscovered animal species."

The target passage parallels the base except for the ambiguity (as in Experiment 1). In the target for the base above, a marine biologist is said to be studying animal life in the Caribbean, using a new oil-drilling platform as a home base, when her attention is caught by a sea turtle with unusual physical features. Although the story states that she realizes she has discovered something important, the specific reason for this importance is left unstated. (The target story stops at this point, and does not parallel the section describing the "alternate" concept in the base.)

Steps were taken to ensure that the two relevant base facts were equated for salience and memory in the passages. First, as a conservative measure, the fact in the structurally nonmatching role was explicitly mentioned multiple (three) times in each base passage, but the structurally matching concept was mentioned only once. Also, a separate control condition in which participants $(n=10)$ answered questions after reading the base and filler passages (but not the targets) revealed no difference in relative recall memory for the facts in the two positions.

If the results of Experiment 1 were due simply to increased semantic activation for ideas mentioned in the base stories, we should see no effect of structural consistency in the present experiment. 
All participants had been exposed to both of the key base facts, and both should be available to support comprehension of the target. In contrast, if structural alignment plays a role in the way the first story influences the second, the role of the information in the passage should have important consequences. According to a structuremapping account, potential inferences would be projected according to corresponding structural positions in the two mental representations. This account thus predicts that target interpretations should reflect the information in the earlier, matching portion of the base.

The test questions again consisted of 2 items per story, making 18 items in total. As in the first experiment, each of the 2 items pertaining to a target passage was consistent with one of the possible interpretations suggested by the key facts in the base. In the example above, for instance, 1 item was consistent with the sea turtle's being an undiscovered species, the other with its being a mutation resulting from pollution. The filler questions again consisted of one correct yes and one correct no item per story. The instruction sets and procedures were identical to those in Experiment 1.

\section{Results and Discussion}

A one-way within-subjects ANOVA confirmed that participants were more likely to respond that a fact had been stated in the target when that fact was consistent with information playing a corresponding role in the base $[F(1,23)=6.25, p<.05]$. That is, participants were significantly more likely to infer the key fact from the base when it was in the same conceptual position as the ambiguous portion of the target. Participants responded affirmatively to structurally corresponding items $58 \%$ of the time, and to noncorresponding items only $31 \%$ of the time. An items analysis using structural correspondence as a within-items factor was also significant $[F(1,3)=$ $46.09, p<.01]$, as was a $\operatorname{Min} F^{\prime}$ analysis over both participants and items $\left[\operatorname{Min} F^{\prime}(1,26)=5.50, p<.05\right]$.

These results show considerable structural sensitivity. Even though all participants had seen the same two relevant concepts, either of which could be used to support target comprehension, they were far more likely to make use of a base element when it corresponded structurally to the ambiguous part of the target story. The results are consistent with the proposal that the inferences were made via structural alignment and projection from the base to the target. We next turn to the question of when the process takes place-during comprehension, or at a later time.

\section{EXPERIMENT 3}

The method used in the first two experiments - asking which facts had been explicitly stated in the target passages - clearly showed structural effects on the participants' final representations of the target stories after reading. However, this approach leaves itself open to questions about the effects of reconstructive memory on the observed results. Our goal has been to determine whether individuals make use of prior analogous instances during the actual interpretation of a new episode. It is also possible, however, that the results we have found are instead due to effects of recall processes during the test phase of our experiments. For instance, participants could simply be remembering the base story rather than the target. Although steps were taken to make this less likely_-including giving a story title with each test item and changing salient details, such as setting and character gender, between base-target pairs - the general similarity between the base-target pairs makes misremembering a real possibility. There is also a chance that participants conflated the two stories, reconstructing a memory that included details from both the base and target passages. Similarly, individuals may have created an abstract representation based on features and structural commonalities of both stories and used this representation rather than the target passages as the basis for their responses.

To address explanations such as these, we used a computer-based reading speed measure that allowed us to examine more closely the participants' processes at the time of encoding the target. The passages from Experiment 1 were modified by adding a sentence to each target that would be consistent with one of the possible interpretations and inconsistent with the other. If the base passage influences processing of the target during online comprehension, participants should show confusion - and therefore slower reading times - when a sentence in the target is inconsistent with the interpretation suggested by the key fact in the base.

It is important here to clarify our use of the reading speed measure and the ways that this use differs from how such measures have been used in prior research. It has been noted that the process of making an inference tends to slow reading and that this may provide one way of examining whether and when inferences are made. For instance, Catrambone (2002) used reading speeds to show that relational commonality between passages could contribute to reminding, as evidenced by slower reading times at likely inference points in a target passage. The present research, on the other hand, concerns the content of participants' inferences and the degree to which that content is incorporated in their representations. To that end, in Experiment 3 we did not examine reading times at the presumed time of the inference (since inferences were expected in all conditions), but rather the reading times for later sentences whose content was either consistent or inconsistent with the predicted inferences.

\section{Method}

Participants. The participants were 20 undergraduate students at Northwestern University who participated either for pay or for class credit.

Materials and Procedure. The materials consisted of modified versions of the set of narrative passages used in Experiment 1. Since reading speeds of the sentences in the passages themselves served as the dependent measure, there were no additional test items. A sentence was added to each of the target passages that was consistent with one of the possible interpretations and inconsistent with the other. All participants read the same target passages, but whether the additional sentence was consistent or inconsistent with base-derived inferences depended upon which version of the base passage had been read.

For instance, consider the target passage described with Experiment 1 , in which it was left ambiguous whether the nephew, George, was fleeing the scene of a crime or coming into town for his uncle's funeral. In the present experiment, the following sentence was added later in the passage: "George's absence from the service was conspicuous, especially since he had been seen around his uncle's estate prior to his death, and the police soon found out about his flight to 
Rio." If the reader interpreted the nephew's trip as fleeing the scene of a crime, this test sentence was reasonably easy to understand. If the trip was understood as the nephew coming to the uncle's funeral, however, the sentence would become not only unexpected, but somewhat incomprehensible. Reading times for this sentence therefore provide a way of assessing which inference was made during online processing of the earlier ambiguous sentence. The base passages were varied between participants such that each individual read one target that was consistent and one that was inconsistent with inferences from the bases that had been read. Across participants, each target passage was read an equal number of times as consistent and inconsistent with the base information.

Participants read the passages from a computer monitor. The stories were presented one sentence at a time, with participants pressing the space bar to advance to the next sentence. The reading time for each sentence was recorded, measured from the time a sentence appeared on the screen until the space bar was pressed. The title of each story remained at the top of the screen while each sentence was read. At the end of each story the screen cleared, and the participant could not proceed to the next passage until the space bar was pressed again.

\section{Results and Discussion}

Reading times for the critical added sentences, which could be either consistent or inconsistent with the basederived inferences, served as the dependent measure. These were calculated as reading times per syllable in order to provide a general metric across sentences. The average per-syllable reading time for a relevant sentence was $137 \mathrm{msec}$ when it was consistent with the expected inference from the base, as compared with $182 \mathrm{msec}$ for the same sentence when it was inconsistent $[F(1,19)=$ $5.16, p<.05]$ - roughly a third again as long. This disparity of $45 \mathrm{msec}$ (over $2 \mathrm{sec}$ total per sentence, on average) represents a sizeable difference. In fact, for 17 of the 20 participants, per-syllable reading times were longer for a critical sentence that was inconsistent with the potential base inferences than for one that was consistent.

These results suggest that the contents and structure of the base passage do influence the comprehension of the target as it is being read. Identical sentences were read significantly more slowly when they conflicted with inferences suggested by an earlier analogous story. This indicates that differences in the relevant portion of the base passages (the only difference between conditions) had a significant influence on the processing of the later target stories during encoding. These effects are important because they cannot be attributed to later artifacts occurring during a recognition test. Of course, these results do not rule out the possibility that additional recognition time effects could have contributed to the findings from Experiments 1 and 2. However, this experiment provides clear evidence that an analogous base passage is capable of exerting an influence on the online comprehension of a target.

\section{GENERAL DISCUSSION}

The results of these experiments support the idea that structural information from a single analogous instance can influence the way in which a new instance is understood, without an individual's deliberation, or perhaps even awareness. In Experiments 1 and 2, participants inter- preted new narrative passages in a way that indicated they were using an earlier passage as a source for inference. The same target passage was interpreted in different ways depending on which base was read, and these interpretations were consistent with inferences from that particular base passage. Furthermore, in open-ended questions, the great majority of participants reported that they were unaware of these inferences having occurred, or even of the ambiguities in the target passages that required resolution. Despite generally recognizing the similarity between the passages, $80 \%$ of the participants asserted that they had not used earlier passages in understanding later ones, and that all of the passages were completely understandable on their own. Experiment 2 further established that this nonintentional inference process involved the alignment of representational structure rather than a simple priming process, since a given assertion had a much greater influence on the target's interpretation when it played a structurally identical role in the base passage. Finally, results from the third experiment showed that these inferences can take place during the actual reading and comprehension of the target passages, rather than simply as a result of later memory processes: Participants took significantly longer to read a sentence in the target when it was inconsistent with an earlier inference from the base. Taken together with the results from the first two experiments, these findings support the conclusion that analogical inference from an earlier passage can influence the representation of a later passage during encoding. These results are consistent with the possibility that analogical inference may occur as part of the fundamental process of assigning meaning to the things we encounter. They also support the idea that the partial representations of events, as those events are being experienced, may be aligned with existing mental representations in a way that activates common structure and allows for the importation of structurally matching information. This finding is important for a number of reasons. First, it acts as an existence proof that processes of structural alignment and inference can act in a way that is much more immediate and nondeliberate than has generally been assumed. Additionally, as a research tool, it allows a well-defined and -investigated system, structuremapping, to be used in understanding a ubiquitous but complex set of processes. More generally, it demonstrates the intricate way in which mental processes interact and may be recruited in support of one another.

Prior work has examined the role of analogy in providing structure to novel experiences, and sometimes in altering the structure of existing representations, but these analogies have generally been explicitly available to the individual during processing and have not been directly related to the general comprehension of the target. Research on schemas has demonstrated the powerful effects that existing knowledge may have on the interpretation of new input, sometimes without apparent participant awareness. However, that research has dealt with more generalized abstract knowledge structures and has left issues of processing underspecified. The literature on narrative text comprehension has addressed the ways in which inferences may be made during reading to support the global 
comprehensibility of a passage, but it cannot easily account for how a seemingly independent passage could influence this process. The present experiments thus bear on several areas of study, including analogy, schemas, and comprehension.

Finally, our findings support the idea that concrete and abstract knowledge representations - such as specific story representations versus plot schemas - may be processed in some of the same ways. This is consistent with the previously mentioned idea of a representational continuum from concreteness to abstraction, and also follows from recent ideas on metaphor comprehension, in which seemingly distinct methods of interpretation-based on either comparison or categorization - may potentially be viewed as utilizing a common alignment process, distinguished only by level of abstraction (Bowdle \& Gentner, 2005; Wolff \& Gentner, 2000).

\section{Constraints on These Effects}

The results of the present experiments show that analogical processes are capable of influencing online text comprehension. However, these findings are based on a relatively small set of experimental materials that were designed to test for such effects. Much work remains to be done to delineate the scope of these kinds of processes. In fact, the present findings are quite surprising, given that a substantial number of studies have shown extremely low rates of spontaneous transfer from single instances (Gick \& Holyoak, 1980, 1983; Holyoak \& Koh, 1987; Keane, 1988; Ross, 1987, 1989). Additionally, many studies have shown that comparison of two or more instances can produce impressive transfer when isolated instances cannot (Catrambone \& Holyoak, 1989; Gentner, Loewenstein, \& Thompson, 2003; Gick \& Holyoak, 1983; Loewenstein, Thompson, \& Gentner, 2003), suggesting that this sort of effect may often require abstracted schemas rather than concrete instance representations. We can look to differences between our experiments and this previous research for clues about possible constraints on our effects.

One potentially important factor is the degree and kind of similarity between the base and target passages. Previous research has established the importance of surface similarity in explicit analogical reminding (Gentner \& Landers, 1985; Gentner et al., 1993; Holyoak \& Koh, 1987), showing a dissociation between the kind of similarity that supports alignment and inferencing (i.e., structural similarity) and the kind that supports memory access to prior instances (i.e., surface similarity-similar characters, objects, and settings). In the present study, the story pairs were high in both structural and surface similarity. Thus, it could be that spontaneous inferencing requires a high degree of surface similarity. However, there is some evidence (Catrambone, 2002; Gentner et al., 1993; Wharton, Holyoak, \& Lange, 1996) that purely structural similarity also contributes to analogical reminding, leaving open the possibility that spontaneous transfer might occur even without surface similarity. We are currently investigating this question.

One significant way in which the present study differs from past research is in the kind of inferences involved.
First, the particular "bridging" inferences required for the interpretation of the ambiguous portions of our target passages were constrained to only a few plausible options. This constraint may have allowed for a more sensitive measure of spontaneous transfer than have prior studies involving more open-ended possibilities for inference. Second, the inferences were rather important to the target story, making them possibly more likely to be mapped across stories than irrelevant details would be.

\section{Resonance Models of Activation}

Our results are consistent with research that has examined the role of general memory processes in the generation of inferences and maintenance of coherence in text, largely focusing on "resonance" models of memory activation (e.g., Gillund \& Shiffrin, 1984; Hintzman, 1986; Ratcliff, 1978). According to resonance models, items in long-term memory may be selectively activated (in parallel) as a function of their featural overlap with the current contents of working memory. These approaches have been successfully used to explain phenomena such as anaphor resolution and reactivation of distant, but relevant, information from earlier in a text (see O'Brien, 1995, for a review). It seems reasonable that these kinds of processes could play a role in the effects found in the present experiments. The high degree of similarity between the source and target passages could have allowed resonance-type activation of the base passage as a whole, or even have supported more localized element-to-element mappings in the course of reading. It is clear, however, that the effects observed in our experiments require more than simple feature-level activation. Our results may best be captured by a model like MAC/FAC (Forbus et al., 1995), which assumes a parallel resonance-like process that finds potential memory matches, followed by a structure-mapping process that aligns (or rejects) these potential matches. This kind of model can capture the fact that the inferences in our study were sensitive to the role that the elements played in the larger relational structure.

\section{Conclusion}

The flow of experiences typically consists of a series of specific, concrete events. We meet specific people, perform particular concrete actions, and notice the tangible instantiated attributes of the specific objects in our surroundings. There are a number of constraints on how these concrete elements are understood and interpreted - such as idiosyncrasies of our perceptual systems or innately defined processes that act on the information we receive - but one of the clearest influences on how new knowledge is acquired is the impact of the rich, structured body of knowledge that individuals already possess. New experiences are filtered through old ones and organized according to schemas abstracted from multiple instances. Beyond this, the present set of experiments has demonstrated our capacity to interpret new information through a surprisingly rapid and nondeliberative structured comparison with a specific prior case. In this way, each moment's concrete content is inextricably linked to events in the past, allowing us to find points of contact and create meaning. 


\section{AUTHOR NOTE}

This research was supported by ONR Grant N00014-02-1-0078 awarded to the second author. We thank Doug Medin, Mark Jung-Beeman, Jason Jameson, and the Language \& Cognition lab group for comments and suggestions. Additional thanks to Kathleen Braun for making everything work. Correspondence relating to this article may be sent to S. B. Day, Department of Psychology, Northwestern University, 2029 Sheridan Road, Evanston, IL 60208 (e-mail: s-day2@northwestern.edu).

Note-This article was accepted by the previous editorial team, when Colin M. MacLeod was Editor.

\section{REFERENCES}

ANDERSON, R. C. (1984). Some reflections on the acquisition of knowledge. Educational Researcher, 13, 5-10.

Anderson, R. C., Reynolds, R. E., Schallert, D. L., \& Goetz, E. T. (1977). Frameworks for comprehending discourse. American Educational Research Journal, 14, 367-381.

ANDERSON, R. C., SPIRO, R. J., \& ANDERSON, M. C. (1978). Schemata as scaffolding for the representation of information in connected discourse. American Educational Research Journal, 15, 433-440.

BLANCHETTE, I., \& DunBAR, K. (2002). Representational change and analogy: How analogical inferences alter representations. Journal of Experimental Psychology: Learning, Memory, \& Cognition, 28, 672-685.

BOWDLE, B. F., \& GENTNER, D. (1997). Informativity and asymmetry in comparisons. Cognitive Psychology, 34, 244-286.

BowdLe, B. F., \& GENTNER, D. (2005). The career of metaphor. Psychological Review, 112, 193-216.

BRANSFORD, J. D., \& JOHNSON, M. K. (1972). Contextual prerequisites for understanding: Some investigations of comprehension and recall. Journal of Verbal Learning \& Verbal Behavior, 11, 717-726.

CATrambone, R. (2002). The effects of surface and structural feature matches on the access of story analogs. Journal of Experimental Psychology: Learning, Memory, \& Cognition, 28, 318-334.

Catrambone, R., \& HolyoAK, K. J. (1989). Overcoming contextual limitations on problem-solving transfer. Journal of Experimental Psychology: Learning, Memory, \& Cognition, 15, 1147-1156.

CHENG, P. W., \& HolyOAK, K. J. (1985). Pragmatic reasoning schemas. Cognitive Psychology, 17, 391-416.

CLEMENT, C. A., \& GenTNER, D. (1991). Systematicity as a selection constraint in analogical mapping. Cognitive Science, 15, 89-132.

Dunbar, K. (1995). How scientists really reason: Scientific reasoning in real-world laboratories. In R. J. Sternberg \& J. E. Davidson (Eds.), The nature of insight (pp. 365-395). Cambridge, MA: MIT Press.

Dunbar, K. (1999). How scientists build models: InVivo Science as a window on the scientific mind. In L. Magnani, N. J. Nersessian, \& P. Thagard (Eds.), Model-based reasoning in scientific discovery (pp. 85-99). New York: Kluwer.

Forbus, K. D., GentNer, D., \& LAW, K. (1995). MAC/FAC: A model of similarity-based retrieval. Cognitive Science, 19, 141-205.

Gentner, D. (1983). Structure-mapping: A theoretical framework for analogy. Cognitive Science, 7, 155-170.

GENTNER, D. (2002). Analogy in scientific discovery: The case of Johannes Kepler. In L. Magnani \& N. J. Nersessian (Eds.), Model-based reasoning: Science, technology, values (pp. 21-39). New York: Kluwer.

GentNer, D. (2003). Analogical reasoning, psychology of. In L. Nadel (Ed.), Encyclopedia of cognitive science (Vol. 1, pp. 106-112). London: Nature Publishing.

Gentner, D., Holyoak, K. J., \& Kokinov, B. N. (Eds.) (2001). The analogical mind: Perspectives from cognitive science. Cambridge, MA: MIT Press.

Gentner, D., \& Landers, R. (1985). Analogical reminding: A good match is hard to find. In Proceedings of the International Conference on Cybernetics and Society (pp. 607-613). New York: IEEE Press.

Gentner, D., Loewenstein, J., \& Thompson, L. (2003). Learning and transfer: A general role for analogical encoding. Journal of Educational Psychology, 95, 393-405.

Gentner, D., \& Markman, A. B. (1997). Structure mapping in analogy and similarity. American Psychologist, 52, 45-56.

Gentner, D., \& MedinA, J. (1998). Similarity and the development of rules. Cognition, 65, 263-297.
Gentner, D., Rattermann, M. J., \& Forbus, K. D. (1993). The roles of similarity in transfer: Separating retrievability from inferential soundness. Cognitive Psychology, 25, 524-575.

Gick, M. L., \& HolyOAK, K. J. (1980). Analogical problem solving. Cognitive Psychology, 12, 306-355.

GICK, M. L., \& HoLYOAK, K. J. (1983). Schema induction and analogical transfer. Cognitive Psychology, 15, 1-38.

GiLlund, G., \& SHIFFRIN, R. M. (1984). A retrieval model for both recognition and recall. Psychological Review, 91, 1-67.

Glenberg, A. M., MEYER, M., \& LindeM, K. (1987). Mental models contribute to foregrounding during text comprehension. Journal of Memory \& Language, 26, 69-83.

Graesser, A. C., Singer, M., \& Trabasso, T. (1994). Constructing inferences during narrative text comprehension. Psychological Review, 101, 371-395.

HiNTZMAN, D. L. (1986). Schema "abstraction" in a multiple-trace memory model. Psychological Review, 93, 411-428.

HoLYOAK, K. J., \& KoH, K. (1987). Surface and structural similarity in analogical transfer. Memory \& Cognition, 15, 332-340.

Holyoak, K. J., \& ThagaRD, P. (1989). Analogical mapping by constraint satisfaction. Cognitive Science, 13, 295-355.

Holyoak, K. J., \& THAGARD, P. (1995). Mental leaps: Analogy in creative thought. Cambridge, MA: MIT Press.

Hummel, J. E., \& HolYOAK, K. J. (1997). Distributed representations of structure: A theory of analogical access and mapping. Psychological Review, 104, 427-466.

KEANE, M. T. (1988). Analogical problem solving. New York: Wiley.

KINTSCH, W. (1988). The role of knowledge in discourse comprehension: A construction-integration model. Psychological Review, 95, 163-182.

KoKINOV, B., \& French, R. M. (2003). Computational models of analogy making. In L. Nadel (Ed.), Encyclopedia of cognitive science (Vol. 1, pp. 113-118). London: Nature Publishing.

Loewenstein, J., Thompson, L., \& Gentner, D. (1999). Analogical encoding facilitates knowledge transfer in negotiation. Psychonomic Bulletin \& Review, 6, 586-597.

LoEWEnstein, J., Thompson, L., \& Gentner, D. (2003). Analogical learning in negotiation teams: Comparing cases promotes learning and transfer. Academy of Management Learning \& Education, 2, 119-127.

Markman, A. B. (1997). Constraints on analogical inference. Cognitive Science, 21, 373-418.

MEDIN, D. L., \& Ross, B. H. (1989). The specific character of abstract thought: Categorization, problem solving, and induction. In R. J. Sternberg (Ed.), Advances in the psychology of human intelligence (Vol. 5, pp. 189-223). Hillsdale, NJ: Erlbaum.

Moreau, C. P., Markman, A. B., \& Lehmann, D. R. (2001). "What is it?" Categorization flexibility and consumers' responses to really new products. Journal of Consumer Research, 27, 489-498.

Novick, L. R. (1988). Analogical transfer, problem similarity, and expertise. Journal of Experimental Psychology: Learning, Memory, \& Cognition, 14, 510-520.

O'BRIEN, E. J. (1995). Automatic components of discourse comprehension. In R. F. Lorch, Jr. \& E. J. O'Brien (Eds.), Sources of coherence in reading (pp. 159-176). Hillsdale, NJ: Erlbaum.

Perrott, D. A., Gentner, D., \& Bodenhausen, G. V. (2005). Resistance is futile: The unwitting insertion of analogical inferences in memory. Psychonomic Bulletin \& Review, 12, 696-702.

RATCLIFF, R. (1978). A theory of memory retrieval. Psychological Review, 85, 59-108.

Ross, B. H. (1984). Remindings and their effects in learning a cognitive skill. Cognitive Psychology, 16, 371-416.

Ross, B. H. (1987). This is like that: The use of earlier problems and the separation of similarity effects. Journal of Experimental Psychology: Learning, Memory, \& Cognition, 13, 629-639.

Ross, B. H. (1989). Remindings in learning and instruction. In S. Vosniadou \& A. Ortony (Eds.), Similarity and analogical reasoning (pp. 438-469). Cambridge: Cambridge University Press.

Ross, B. H., \& Bradshaw, G. L. (1994). Encoding effects of remindings. Memory \& Cognition, 22, 591-605.

RumelHART, D. E., \& ORTONY, A. (1977). The representation of knowledge in memory. In R. C. Anderson, R. J. Spiro, \& W. E. Montague 
(Eds.), Schooling and the acquisition of knowledge (pp. 99-135). Hillsdale, NJ: Erlbaum.

Spellman, B. A., \& HolyoaK, K. J. (1996). Pragmatics in analogical mapping. Cognitive Psychology, 31, 307-346.

Steffensen, M. S., JoAG-Dev, C., \& Anderson, R. C. (1979). A crosscultural perspective on reading comprehension. Reading Research Quarterly, 15, 10-29.
Thagard, P. (1992). Conceptual revolutions. Princeton, NJ: Princeton University Press.

Wharton, C. M., Holyoak, K. J., \& LAnge, T. E. (1996). Remote analogical reminding. Memory \& Cognition, 24, 629-643.

WolfF, P., \& GentNER, D. (2000). Evidence for role-neutral initial processing of metaphors. Journal of Experimental Psychology: Learning, Memory, \& Cognition, 26, 529-541.

\section{APPENDIX A \\ Sample Materials From Experiment 1}

\section{Bases A \& B}

When the widow-heiress Margaret Haverty died suddenly, there were some who suspected foul play.

Ms. Haverty, who was quite wealthy, had lived in Amsterdam for the last 10 years, since the loss of her husband.

Her death received quite a bit of public attention, and was even reported in the international media.

(Version A) As soon as the news of her death was announced, her niece Helena mysteriously booked a flight from Amsterdam, where she also lived, off to Naples.

(Version B) As soon as the news of her death was announced, her niece Helena respectfully booked a flight from Naples, where she lived, to Amsterdam for the funeral.

(Version A) Helena had known about her aunt's wealth since childhood.

(Version B) Helena had been close to her aunt since childhood.

The funeral, which was held 2 days later, was enormous, and all of the relatives who were in town attended, along with many who were just curious to see the spectacle.

After the funeral, Ms. Haverty's lawyers revealed the contents of her will, which left the bulk of her estate to her niece Helena.

There were many other relatives who were surprised and upset by this, particularly Haverty's two children, who had expected to inherit everything.

The police suspected that money may have been a motive for murder, and they opened an investigation into Haverty's death.

They were especially attentive to any suspicious behavior during the days immediately before and after her demise.

\section{Target}

The death of the wealthy old Alexander van Houton was abrupt and unexpected, and there were rumors that it might not be of natural causes.

Mr. van Houton had moved to a large family estate in a warm climate when his wife died several years earlier, and it was there that his body was discovered by servants.

His death sparked widespread interest, and even received attention in the overseas press.

Surprisingly, when the news of the death was released, Mr. van Houton's nephew George immediately bought a ticket and flew to Rio de Janeiro.

George had known his uncle since childhood.

Relatives and friends turned out in great numbers to attend the funeral, which was quite lavish.

Later that same day, the executor of Mr. van Houton's estate publicly read his will-his nephew George had inherited almost everything.

This came as an unpleasant shock to many, especially van Houton's son and daughter, who had thought that they would be left with a fortune.

The authorities believed that someone might have killed Alexander van Houton because they had expected to inherit money, and an investigation into the death was begun.

They immediately began searching the scene for clues, and interviewing anyone who might be able to provide useful information.

\section{Test Items}

George had been in the same city as his uncle, but left town when the death was announced. George was among those who came into town for Alexander van Houton's funeral. 


\section{APPENDIX B}

\section{Sample Base and Target Passages From Experiment 2}

\section{Base Passage: "Jungle Expedition"}

Dr. Paul Chapman, a herpetologist, was on an expedition to study the indigenous animal life in the Camisean Jungle in Peru. The recent development of an industrial refinery nearby had made the area much more accessible for travel, and Dr. Chapman was one of the first scientists to thoroughly explore the region. While working his way through the dense vegetation one afternoon, he noticed something that immediately caught his attention, and the longer he looked the more thrilled he became. It clearly resembled the horned wood lizard, which is not uncommon to the region, but its coloration was far darker than he had ever seen, and its limbs were much more slender, appearing almost fragile. Dr. Chapman realized immediately that what he was seeing was (A) an undiscovered species / (B) a physical deformity resulting from pollution from the refinery, and he knew that he needed to take it back to his base camp for a more thorough evaluation. Closer study confirmed his initial impression, and he understood that he had discovered something very important. When he returned to the United States several months later, the report of his findings had a huge impact on the scientific community.

As a result of the notoriety from his discovery, Chapman soon received an invitation from a prestigious institution asking him to work on a different kind of project. He would work in the institution's offices in Washington, D.C., and would be in charge of deploying various field teams to (A) investigate the impact of pollution on animal growth and development / (B) search for previously undiscovered animal species. In addition to administrating the teams' operations, he would also supervise the evaluation of the evidence they returned. Dr. Chapman took the issue of (A) the impact of pollution / (B) investigating new species very seriously, and was remarkably diligent and conscientious in his work. Several months later, as the project was drawing to a close, his colleagues decided to honor him for his important contribution. They emphasized the impact that his work on (A) pollution / (B) species discovery would have for future generations. True to his restless and curious nature, however, Dr. Chapman was never content to rest on his prior accomplishments, and he was soon making arrangements for a new expedition to the African grasslands.

\section{Target Passage: "Marine Biology"}

Mary Sutcliffe was a renowned marine biologist, and currently she was studying the animal life in the waters of the Caribbean, about 600 miles northeast of the Dominican Republic. A large offshore platform for oil drilling had been constructed in the area a few months ago, and it provided a convenient base from which to explore an otherwise isolated region. Because of this, Dr. Sutcliffe had the opportunity to conduct research of the area that was far more thorough and comprehensive than previously had been possible. While making a dive one morning, she saw something that immediately caught her eye, and her attention grew as she looked more closely. What she saw generally looked like a hawksbill sea turtle, but unusual reddish patches on its shell gave it a weird, mottled appearance, and its eyes seemed pale and opaque. Dr. Sutcliffe felt her heart race as she realized what she had found, and she gently brought the animal back to her boat, and then to her base laboratory for closer study. Further evaluation verified her intuitions, and she knew that she needed to inform her fellow scientists of this discovery immediately so that appropriate action could be taken. Indeed, the reports of her finding created quite a stir among the biological community in general. No one doubted the validity of her research because of her impeccable record as an honest and meticulous scientist. She had already received attention for her research on migration patterns of the humpback whale and predatory behaviors of the cuttlefish. As soon as she had finished her current project, Dr. Sutcliffe heard about a voyage to the Arctic Ocean to study the narwhal, and she immediately signed on for the trip.

Note-Differences between the two base versions (A and B) are in bold.

(Manuscript received November 10, 2003; revision accepted for publication August 23, 2005.) 